\title{
Self-Consistent $\mathbf{S}$ Matrix with Regge Asymptotic Behavior
}

\author{
Geoffrey F. Chew* \\ CERN, Geneva, Switzerland \\ (Received 31 August 1962)
}

\begin{abstract}
A general discussion is given of the formulation of self-consistent equations for two-body strong-interaction $S$ matrix elements, on the assumption that all poles are of the Regge type and that asymptotic behavior is controlled by these poles. A tentative set of specific equations based on the strip approximation is presented and illustrated by a discussion of the $\pi \pi$ problem. In these equations Regge trajectories and residues for one channel are determined by the top-level Regge poles of the crossed channels. One parameter, the width of the strip, appears in the equations but it is argued that this parameter may be fixed by the principle of maximum strength.
\end{abstract}

\section{INTRODUCTION}

$T$ HERE have been many attempts to formulate "bootstrap" equations within the framework of the analytically continued strong-interaction $S$ matrix, in order to generate certain of the observed particles from the assumed existence of others. ${ }^{1-5} \mathrm{Few}$ of these attempts, however, have employed the assumption of Regge asymptotic behavior and none has attempted to exploit the full consequences of such behavior. The purpose of this paper is twofold: (a) to discuss the general impact of the Regge-pole assumption on dynamical equations for two-body $S$ matrix elements; (b) to illustrate some of the essential points with a crude treatment (strip approximation) of the $\pi \pi$ problem, where the goal is to produce the top-level Regge trajectories with $B=S=0, G=+1, I=0,1,2$, in terms of the assumed existence of the pion.

By the Regge-pole assumption we mean the following: All poles of the strong-interaction $S$ matrix can be continued in angular momentum, all retreating to the left half of the $J$ complex plane $(\operatorname{Re} J<0)$ for sufficiently large (negative) energies. ${ }^{6}$ It has been emphasized previously that such an assumption corresponds to all baryons and mesons being composite, with no internal point structure; more precisely, there are no arbitrary subtraction terms in the Mandelstam representation. According to the Sommerfeld-Watson transform connecting $A(s, J)$ to $A(s, t){ }^{7}$ the asymptotic behavior in the variable $t$ is $\left\langle t^{J_{\max }(s)}\right.$, where $J_{\max }(s)$ is the position of the rightmost singularity in the $J$ plane. ${ }^{8}$ If for some range of $s, \operatorname{Re} J_{\max }(s)<0$, the amplitude here vanishes sufficiently rapidly as $t \rightarrow \infty$ so that no arbitrary sub-

* Permanent address: Department of Physics and Lawrence Radiation Laboratory, University of California, Berkeley, California.

${ }^{1}$ G. F. Chew and S. Mandelstam, Phys. Rev. 119, 467 (1960); Nuovo Cimento 19, 752 (1961).

${ }_{2}^{2}$ M. Cini and S. Fubini, Ann. Phys. (N. Y.) 3, 358 (1960).

3 A. V. Efremov, V. A. Meshcheryakov, D. V. Shirkov, and H. Y. Izu, Nucl. Phys. 22, 202 (1961).

${ }^{4}$ G. F. Chew and S. C. Frautschi, Phys. Rev. 123, 1478 (1961). $5 \mathrm{D}$. Wong, Phys. Rev. 126, 1220 (1962).

${ }^{6}$ G. F. Chew and S. C. Frautschi, Phys. Rev. Letters 7, 394 (1961) ; 8, 41 (1962).

${ }^{7}$ We shall ignore throughout complications due to spin, but the essential points of the paper are presumed to be general.

${ }^{8}$ A. Sommerfeld, Partial Differential Equations in Physics (Academic Press Inc., New York, 1949), p. 279. tractions in $t$ are allowed; then by analytic continuation in $s$, as discussed by Froissart, subtraction terms everywhere else are determined. ${ }^{9}$ Chew, Frautschi, and Mandelstam have shown explicitly how to make this analytic continuation when the controlling singularity is a pole,$^{10}$ and, according to the equations to be derived here, the only singularities extending into the right-half $J$ plane are, in fact, simple poles.

The asymptotic behavior, then, of the amplitude $A(s, t, u)$ describing the three processes

$$
\begin{array}{rll}
\text { I. } & a+b \rightarrow c+d, & \text { energy squared }=s, \\
\text { II. } & a+\bar{c} \rightarrow \bar{b}+d, & \text { energy squared }=t, \\
\text { III. } & a+\bar{d} \rightarrow \bar{b}+c, & \text { energy squared }=u,
\end{array}
$$

will be taken as that of Fig. 1. Here $\alpha^{\mathrm{I}}(s)$ is the rightmost Regge trajectory with the quantum numbers of channel I and $\alpha^{\mathrm{II}}(t), \alpha^{\mathrm{III}}(u)$ are the rightmost trajectories for channels II and III. If for certain quantum numbers more than one trajectory extends into the

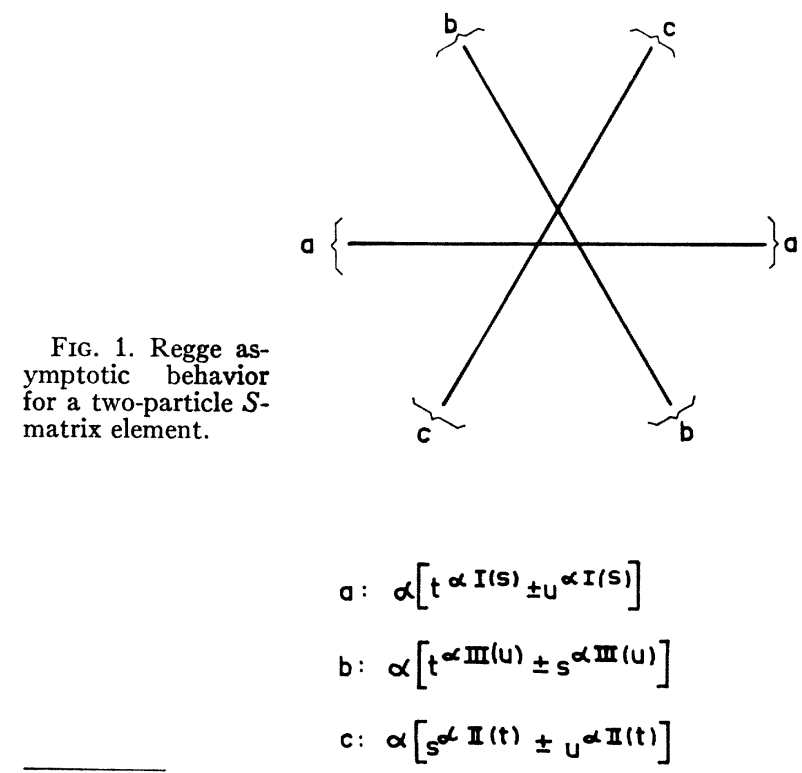

'M. Froissart, Phys. Rev. 123, 1053 (1961).

${ }^{10} \mathrm{G}$. F. Chew, S. C. Frautschi, and S. Mandelstam, Phys. Rev. 126, 1204 (1962). 


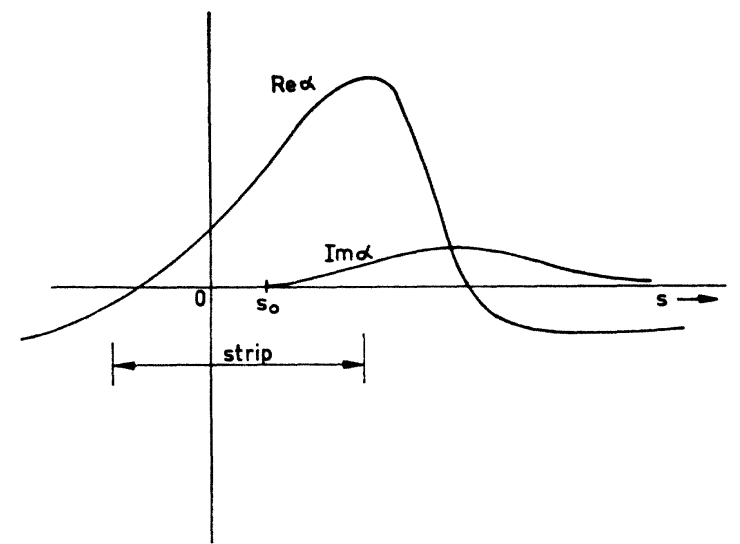

FIG. 2. A typical high-level Regge trajectory corresponding to strongly attractive forces.

right-half $J$ plane one simply takes the appropriate sum of powers, weighted with the Regge-pole residues. (When all trajectories for a particular set of quantum numbers happen to remain in the left-half plane, the amplitude vanishes asymptotically in the corresponding direction in $s, t, u$ space.) The choice of the plus or minus sign depends on the $J$ signature of the trajectory, as has been explained by several authors. ${ }^{11}$

What is the general nature of the trajectories that succeed in reaching (or closely approaching) the righthalf $J$ plane? It has been shown by Barut and Zwanziger and by Taylor that each trajectory $\alpha(s)$ is a real analytic function and that, if there is no intersection with another trajectory, $\alpha(s)$ has only the right-hand cut extending from the physical threshold $s_{0}$ to infinity. ${ }^{12}$ If we assume further that $\alpha(s)$ is everywhere bounded (see reference 15) we may write the dispersion relation

$$
\alpha(s)=\alpha(\infty)=\frac{1}{\pi} \int_{s_{0}}^{\infty} d s^{\prime} \frac{\operatorname{Im} \alpha\left(s^{\prime}\right)}{s^{\prime}-s}
$$

suggested by Singh. ${ }^{13}$ It has been proved by Barut and Zwanziger ${ }^{12}$ that

$$
\operatorname{Im} \alpha \underset{s \rightarrow s_{0}}{\propto}\left(s-s_{0}\right)^{\alpha\left(s_{0}\right)+1 / 2}, \quad \alpha\left(s_{0}\right)>-\frac{1}{2},
$$

and it is plausible for trajectories that reach the righthalf $J$ plane that

$$
\operatorname{Im} \alpha \geq 0
$$

throughout the $s$ physical region. The latter property has so far been proved only for potential scattering but seems likely to be general since it arises from the physical requirement that resonances decay rather than grow with time. ${ }^{14}$ Combining all these features, which

${ }^{11}$ See, for example, S. Frautschi, M. Gell-Mann, and F. Zachariasen, Phys. Rev. 126, 2204 (1962); and E. Squires, Nuovo Cimento 25, 242 (1962).

${ }^{12}$ A. Barut and D. Zwanziger, Phys. Rev. 127, 974 (1962)

J. R. Taylor, ibid. 127, 2257 (1962).

${ }^{13}$ V. Singh, Phys. Rev. 127, 632 (1962).

1s T. Regge, Nuovo Cimento 18, 947 (1960). seem consistent with the equations to be derived, we conclude that the general form of an interesting trajectory should be as shown in Fig. $2 .^{15}$

This figure gives a precise meaning to the strip concept introduced by Chew and Frautschi. ${ }^{4}$ In the finite interval for which $\operatorname{Re} \alpha(s)>0$ with $\operatorname{Im} \alpha \leqslant 1$ the trajectory dominates the amplitude. For $s>0$ there will be bound state or resonance poles when $\operatorname{Re} \alpha$ intersects physical integers (with $d \operatorname{Re} \alpha / d s>0$ ), and for $s<0$ we are in the physical region of the crossed channels where the high $t$ (or $u$ ) behavior is controlled by the trajectory. For large values of $s$, either positive or negative, the trajectory recedes into the left-half $J$ plane where its effect becomes submerged among the other $J$ singularities. Isolated trajectories are dominant only within strips in the Mandelstam diagram and the "strip approximation" may be restated as the representation of the full amplitude by those top-ranking Regge poles that reach (or closely approach) the right-half $J$ plane.

The width of a strip can be estimated in terms of $\alpha(s)$ at $s=0$ because in (I.1) the principal value integral will vanish near the maximum in $\operatorname{Im} \alpha$, this maximum occurring near

$$
\begin{aligned}
\bar{s}=\int_{s_{0}}^{\infty} d s^{\prime} \frac{\operatorname{Im} \alpha\left(s^{\prime}\right)}{s^{\prime}} / \int_{s_{0}}^{\infty} d s^{\prime} \frac{\operatorname{Im} \alpha\left(s^{\prime}\right)}{s^{\prime 2}} & \\
= & \frac{\alpha(0)-\alpha(\infty)}{\alpha^{\prime}(0)} .
\end{aligned}
$$

The Froissart limit tells us that $\alpha(0) \leqslant 1^{9}$ and Gribov has shown that $\alpha(\infty) \geq-1 .^{16}$ Thus the width of the strip is $\leqslant(d \alpha / d s)^{-1}$ at $s=0$, i.e., of the order $1 \mathrm{GeV}^{2}$ in typical cases. ${ }^{17}$ All stable and metastable particles correspondingly are expected to have masses $\leqslant 1 \mathrm{GeV}$ and the forward and backward peaks in the crossed channels are confined to momentum transfers of this same order of magnitude. The absence of large mass (energy) ratios may be traced to the lack of arbitrary parameters, the most characteristic feature of our strong interaction theory.

Our problem, of course, is to calculate the trajectories (and residues) of the top-ranking Regge poles across the strips. Could this be done we would have explained almost everything known about strong interactions. But what must be the input of the calculation? This is a

15 Note that for $s<s_{0}$ all derivatives are positive, as emphasized by Singh in reference 13. It is seen in what follows that we actually only depend on the behavior of Fig. 2 for negative $s$. If, in fact, $\operatorname{Re} \alpha(s)$ and $\operatorname{Im} \alpha(s)$ both increase as $s \rightarrow+\infty$, our approach should still be valid. The right-hand boundary of the strip is then determined by the point where $\operatorname{Im} \alpha \gtrsim 1$ and resonances become so broad as to be unrecognizable.

${ }^{16} \mathrm{~V}$. N. Gribov, in Proceedings of the Eleventh High-Energy Conference, Geneva, July, 1962 (CERN, Geneva, 1962).

${ }^{17}$ A natural energy unit for strong interaction physics is the inverse slope of the Pomeranchuk trajectory at zero energy. This slope happens to be close to $1 \mathrm{GeV}^{-2}$ and, as discussed in reference 6 , other high ranking trajectory slopes seem of the same order of magnitude. 
major puzzle since, as noted above, no arbitrary subtraction terms are permitted. More generally one may say that the problem is to calculate the $S$ matrix on the basis of unitarity and analyticity without an $a$ priori knowledge of its dimensionality; to know the dimensions would be already to know which trajectories reach the right-half $J$ plane to produce particles. No program has yet been put forward to generate in a systematic way the full dimensionality of the $S$ matrix. (Presumably such a program would have to explain, among other things, the strong interaction conservation laws.) However, many less ambitious calculations are being attempted, where the existence of certain particles and conservation laws are assumed and one attempts to predict other particles (i.e., trajectories) with quantum numbers arising from combinations. A typical problem of this nature is to start with a knowledge of the existence of the pion and to attempt the calculation of Regge trajectories having the quantum numbers of two-pion systems.

The confusing element here is the redundancy of the input. When one knows that the isotopic spin of the pion is 1 , with $B=S=0$ and $G=-1$, that its angular momentum is zero and its parity odd and also that no other particles of such small mass exist, one has inserted an enormous amount of information, most, if not all, of which should be generated in the exact global solution by our general $S$-matrix principles. At the same time we are forced for practical reasons to neglect or mistreat many parts of the $S$ matrix. It is a dubious proposition, therefore, to strive for a mathematically consistent set of equations until the strong interaction problem is attacked on a global scale. The most we can hope for at present is to find approximately self-consistent solutions, appropriate to a localized portion of the $S$ matrix. Mathematicians throw up their hands at such an illdefined task, but it seems possible, nevertheless, to achieve rough results with physical content. The following section deals with general notions that may be useful in this connection.

\section{QUALITATIVE CONSIDERATIONS}

Almost all calculations undertaken to date have been based on two-body $S$-matrix elements. Is it reasonable to hope for real progress within such a restricted framework? We believe the answer to be affirmative, although the ultimate extent of progress to be expected is unclear. Our optimism is based on the absence of arbitrary dimensionless constants in the strong interaction scheme. The natural unit of mass, as discussed above, is $\sim 1 \mathrm{GeV}$ and without small or large dimensionless parameters all stable and metastable particle masses ought to be of this order of magnitude. The consequence should be that normally a resonance decays principally into two particles; few resonances are sufficiently massive to prefer decay into three or more particles. This argument has substantial experimental support if, in classifying decay products, we include metastable particles, and extension of the definition of the $S$ matrix to unstable particles is now being vigorously studied. ${ }^{18}$ When such is accomplished it seems reasonable to hope that an understanding of the two-body problem suffices for many important purposes. ${ }^{19}$

An objection may be raised that at high energies production multiplicities are known to be large. That is true, but it appears that the bulk of high-energy phenomena is to be understood through a prolongation of Regge trajectories to the region of small negative $s$, in the sense of Fig. 2. Thus, an understanding of lowenergy particles and resonances implies an understanding also of high-energy phenomena, because of the trajectory for small negative $s$ can be calculated by analytic continuation if it is known for positive $s$ across the strip. (See Sec. V.) A pessimist could invert this reasoning, of course, and argue that we should not expect to calculate the properties of particles and resonances without first acquiring a grasp of the manybody problem. There seems no way to resolve such questions except by actually trying calculations. As noted above, a clear mathematical characterization of the problem does not yet appear possible. We proceed then, confining our attention to $S$-matrix elements of the type $a+b \rightarrow c+d$.

Our goal is to approximate with reasonable accuracy the amplitude $A(s, t, u)$ in the strip regions by superposing those Regge poles (believed small in number) that have the relevant quantum numbers and that extend into or closely approach the right-half $J$ plane. Outside the strips the situation is presumed to be too complicated to consider at present. How are we to calculate the top-ranking Regge trajectories? The simplest method seems to be the $N / D$ technique, appropriately modified to include the inelastic region and Regge asymptotic behavior. One derives linear integral equations for $N(s, J)$ and $D(s, J)$ with kernels analytic in $J$, the $s$-channel Regge-pole trajectories then being given by $D(s, \alpha(s))=0$. The $t$ - and $u$-channel trajectories are similarly obtained. The basic deficiency of previous $N / D$ calculations was the incorrect treatment of asymptotic behavior both on the right and left. It seems essential, as discussed in the next section, to correlate these two regions in the sense of Fig. 1 if oneis toavoid confusion about the number of arbitrary parameters.

The calculation of the kernel in the $N / D$ integral equation is based on crossing, that is, analytic continua-

${ }_{18} \mathrm{~J}$. Ball, W. Frazer, and M. Nauenberg, Phys. Rev. 128, 478 (1962) ; H. Stapp, Lawrence Radiation Laboratory Report, 1962 (unpublished); D. Zwanziger, University of California Physics Department Report, 1962 (unpublished).

${ }_{10}$ It should be realized that the currently employed continuation in angular momentum is essentially a two-body continuation. If it can be defined for multiparticle systems, as the optimists believe, it will be by dividing each system into two groups of particles, each group having definite physical angular momentum, and continuing in the relative angular momentum between the groups. There may be alternative methods of continuation possible but the one currently under discussion is based on such a two-body decomposition. 
tion to the $s$-channel physical region from the $t$ and $u$ channels and vice versa. Previously this continuation was plagued by divergent polynomial expansions, but such troubles appear to be removed by the assumption of Regge asymptotics.

Considerable effort has been expended in studying the iterative procedure of Mandelstam as an alternative to the $N / D$ technique. ${ }^{4,10,20}$ As far as the author is concerned the motivation was that he did not understand previously how, otherwise, to formulate the strip approximation. (Also, the problem of threshold behavior has sometimes been awkward in $N / D$.) The formulation proposed here in terms of Regge poles seems reasonably well suited to $N / D$, although it could no doubt be implemented through the iteration procedure. Which of these two techniques turns out the more effective remains to be seen. Perhaps a completely different approach is destined to make both obsolete, but this paper considers the problem from the $N / D$ point of view.

In summary, then, the program is to assume the asymptotic behavior of Fig. 1 , and by the $N / D$ method to generate the Regge trajectories for each channel from the top-level trajectories for the two crossed channels. There will be no subtraction constants or CDD poles in the $N / D$ equations and in principle, therefore, no free parameters in the final result. We shall see, however, that some arbitrariness arises in making the transition across the strip boundary, i.e., from the low-energy resonance region to the high-energy domain where multiple production dominates. Our approach does not seem adequate to describe this transition correctly. Fortunately, we can fall back for assistance on the principle of maximum strength [the requirement that for the Pomeranchuk trajectory $\alpha(0)=1]$. This principle presumably constitutes redundant information in a bootstrap calculation, where the existence of one or more particles is assumed at the beginning, but it is available to resolve ambiguities associated with inadequate approximation procedures.

\section{A MODIFIED SET OF $N / D$ EQUATIONS}

On the basis of the asymptotic behavior of $A(s, t, u)$ indicated in Fig. 1 one easily deduces that

$$
A_{l}(s) \underset{\sigma \rightarrow+\infty}{\propto} s^{\alpha \mathrm{II}, \mathrm{III}(0)-1} / \ln s .
$$

The limit as $s \rightarrow-\infty$ is more complicated and is considered elsewhere; here we merely remark that if $\alpha^{\mathrm{II}}(t)$ or $\alpha^{\operatorname{III}}(u)$ exceed unity over any interval the behavior as $s \rightarrow-\infty$ is sufficiently pathological as to preclude calculational procedures where one explicitly integrates to infinity on the left. The behavior on the right is better than that on the left because of the Froissart limit $\alpha(x) \leqslant 1, x \leqslant 0$. We now develop $N / D$ integral equations restricted to the physical (right-hand) cut. As usual, we

${ }^{20} \mathrm{P}$. Burke and C. Tate, in Proceedings of the Eleventh HighEnergy Conference, Geneva, July, 1962 (CERN, Geneva, 1962). consider the function

$$
B_{l}(s)=q_{s}^{-2 l} A_{l}(s) \underset{8 \rightarrow+\infty}{\propto} s^{\alpha \mathrm{II}, \mathrm{III}(0)-1} / s^{l} \ln s .
$$

Let us denote the total contribution to $B_{l}(s)$ from all the high-level Regge poles in channels II and III by $B_{l}{ }^{P}(s)$, where from $B_{l}{ }^{P}(s)$ we have removed the cut in $s$ over the strip region $0<s<s_{1}, s_{1}$ being the point above which $B_{l}(s)$ is dominated by $B_{l} P(s)$. A procedure for removing the cut is shown in the next section; it does not alter the asymptotic behavior of $B_{l}{ }^{P}(s)$ nor does it appreciably alter the discontinuity of $B_{l} P(s)$ across the unphysical (left-hand) cuts. The reason is that these unphysical discontinuities are dominated by the particles (i.e. the poles in $t$ and $u$ ) of the crossed channels and these poles are not affected when the $s$ cut is pushed out a finite distance. The alteration mainly chops out spurious nonresonant high-l components in channels II and III.

Now, since $B_{l} P(s)$ is real for $0<s<s_{1}$ and since the discontinuity of $B_{l}(s)$ across the unphysical cuts as well as across the physical cut for $s>s_{1}$ is approximately equal to that of $B_{l}{ }^{P}(s),{ }^{21}$ we may write as the basic equation of the (new) strip approximation

$$
B_{l}(s)=B_{l} P(s)+\frac{1}{\pi} \int_{s_{0}}^{s_{1}} d s^{\prime} \frac{\operatorname{Im} B_{l}\left(s^{\prime}\right)}{s^{\prime}-s}
$$

if $s_{0}$ is the physical threshold, and note that the correct asymptotic behavior (III.2), or, more precisely

$$
B_{l}(s) \underset{s \rightarrow \infty}{\longrightarrow} B_{l}^{P}(s)
$$

is immediately guaranteed for $l<\alpha^{\mathrm{II}, \mathrm{III}}(0)$. Since the $N / D$ equations to be developed on the basis of (III.3) can be analytically continued in $l$ we hope that the asymptotic behavior continues to be correct for larger $l$. In any event, the self-consistency problem only involves the lower $l$ range, as shown in Sec. IV.

In our modified $N / D$ approach we cause $N_{l}(s)$ to carry all the singularities of $B_{l}{ }^{P}(s)$, that is, the physical cut from $s_{1}$ to $\infty$ as well as the unphysical cuts. Our new $D_{l}(s)$ has a discontinuity only between $s_{0}$ and $s_{1}$ and is normalized to unity at infinite $s$. Thus, writing

$$
B_{l}(s)=N_{l}(s) / D_{l}(s)
$$

we have

$$
D_{l}(s)=1+\frac{1}{\pi} \int_{s_{0}}^{s_{1}} d s^{\prime} \frac{\operatorname{Im} D_{l}\left(s^{\prime}\right)}{s^{\prime}-s} .
$$

Instead of expressing $N_{l}(s)$ as an integral over its cuts, we write ${ }^{22}$

\footnotetext{
${ }^{21}$ Remember that the poles in $t$ and $u$ are completely contained in the terms from which $B_{l} P^{P}(s)$ has been projected.

${ }^{22} \mathrm{~A}$ similar equation has been derived by J. L. Uretsky, Phys. Rev. 123, 1459 (1961). The author first learned of this type of equation from S. Mandelstam (private communication, 1959).
} 
$N_{l}(s)=B_{l}^{P}(s) D_{l}(s)$

$$
-\frac{1}{\pi} \int_{s_{0}}^{s_{1}} d s^{\prime} \frac{\operatorname{Im}\left\{B_{l}^{P}\left(s^{\prime}\right) D_{l}\left(s^{\prime}\right)\right\}}{s^{\prime}-s}
$$

an expression with the required discontinuities and approaching $B_{l}^{P}(s)$ at infinity, at least for $l<\alpha^{\mathrm{II}, \mathrm{III}}(0)$. Since, by construction, $B_{l}{ }^{P}(s)$ is real in the interval between $s_{0}$ and $s_{1}$ we may rewrite (III.7), with the aid of (III.6), as

$$
\begin{aligned}
N_{l}(s)=B_{l} P(s) & \\
& -\frac{1}{\pi} \int_{s_{0}}^{s_{1}} d s^{\prime} \frac{B_{l}{ }^{P}\left(s^{\prime}\right)-B_{l}{ }^{P}(s)}{s^{\prime}-s} \operatorname{Im} D_{l}\left(s^{\prime}\right) .
\end{aligned}
$$

The final step is to use the unitarity condition in order to obtain an expression for $\operatorname{Im} D_{l}(s)$ in the interval $s_{0}$ to $s_{1}$. Our system of equations becomes closed if we employ the two-body relation

or

$$
\operatorname{Im} B_{l}(s)=B_{l}^{*}(s) \rho_{l}(s) B_{l}(s)
$$

$$
\operatorname{Im} D_{l}(s)=-\rho_{l}(s) N_{l}(s),
$$

where $\rho_{l}(s)$ is the usual phase-space factor, but one must ask if this equation is adequate for the entire interval. In other words, is there important production of manybody systems before one reaches the asymptotic region? As stated above we believe that if all two-body channels are included - with metastable as well as stable particles - then residual multiparticle production should be small for $s<s_{1}$. Now for an arbitrary finite number of stable two-body channels the matrix generalization of the above equations is straightforward, ${ }^{23}$ and recent work indicates that unstable particles can be brought into the same framework. ${ }^{18} \mathrm{We}$ are, therefore, optimistic about the reliability of an eventual closed system of two-body equations. We are not so sanguine about the quantitative adequacy of a one-channel approximation, ${ }^{24}$ but because of its simplicity such an approximation deserves first consideration.

Substituting (III.10) into (III.8) we obtain our final equation

$$
\begin{aligned}
N_{l}(s)= & B_{l} P(s) \\
& +\frac{1}{\pi} \int_{s_{0}}^{s_{1}} d s^{\prime} \frac{B_{l}{ }^{P}\left(s^{\prime}\right)-B_{l}{ }^{P}(s)}{s^{\prime}-s} \rho_{l}\left(s^{\prime}\right) N_{l}\left(s^{\prime}\right),
\end{aligned}
$$

a linear integral equation uniquely determining $N_{l}(s)$ in terms of $B_{l}{ }^{P}(s)$ if the latter is finite throughout the interval $s_{0} \leqslant s \leqslant s_{1}$. Actually, as a result of the cut displacement, the function $B_{l}{ }^{P}(s)$ may be logarithmically

${ }^{23}$ J. D. Bjorken, Phys. Rev. Letters 4, 473 (1960); S. Mandelstam (private communication, 1959).

${ }^{24}$ F. Zachariasen and C. Zemach, Phys. Rev. 128, 849 (1962), have shown, for example, that the $\pi \omega$ as well as the $\pi \pi$ channel is important in discussing the properties of the $\rho$. infinite at the uppr limit $s=s_{1}$ :

$$
B_{l}^{P}(s) \underset{s \rightarrow s_{1}}{\longrightarrow}-(1 / \pi) \operatorname{Im} B_{l}^{P}\left(s_{1}\right) \ln \left(s_{1}-s\right) .
$$

Equation (III.11) is therefore singular if $\operatorname{Im} B_{l} P\left(s_{1}\right) \neq 0$, but only marginally so, and can be shown to possess a unique solution as long as

$$
0 \leqslant \rho_{l}\left(s_{1}\right) \operatorname{Im} B_{l}^{P}\left(s_{1}\right) \leqslant 1 .
$$

This latter condition is not an explicit consequence of our equations but follows from the general unitarity limitation on partial wave amplitudes if one remembers that, in our strip approximation, $\operatorname{Im} B_{l}(s)=\operatorname{Im} B_{l}{ }^{P}(s)$ for $s \geq s_{1}$. If (III.13) is not satisfied our $N / D$ equations have no solution, as can be seen from (III.3) where the left-hand side is bounded by elastic unitarity as $s \rightarrow s_{1}$ from below. Consequently, the logarithmically infinite parts of the two terms on the right of (III.3) must cancel, and this can only happen if (III.13) is satisfied since $\operatorname{Im} B_{l}(s)$ is similarly bounded (by elastic unitarity) as $s \rightarrow s_{1}$ from below.

It is not difficult to show that as $s \rightarrow s_{1}$ both $N_{l}(s)$ and $D_{l}(s)$ behave

where

$$
\propto\left(s_{1}-s\right)^{-\delta_{l}\left(s_{1}\right) / \pi},
$$

$$
\delta_{l}\left(s_{1}\right)=\sin ^{-1}\left[\rho_{l}\left(s_{1}\right) \operatorname{Im} B_{l} P\left(s_{1}\right)\right]^{1 / 2},
$$

and, correspondingly, that $\operatorname{Im} B_{l}(s)$ is continuous across the boundary at $s=s_{1}$. The singularity in our equation, therefore, plays two physically useful roles: First, it requires unitarity in the inelastic region, at least in the form (III.13). Second, it leads to a smooth connection between the two-body and the multiple production regions so far as cross sections are concerned.

In a forthcoming paper, (III.11) will be transformed into a nonsingular (Fredholm) equation; it then follows that in the complex $l$ domain where $B_{l} P(s)$ is holomorphic the functions $N_{l}(s)$ and $D_{l}(s)$ are analytic (except for possible fixed poles in $l$ that cancel in $N / D$ ) and $B_{l}(s)$ correspondingly meromorphic with Regge poles at the zeros of $D_{l}(s)$. Therefore, it is now necessary to consider the construction of the function $B_{l}{ }^{P}(s){ }^{25}$

\section{CONSTRUCTION OF $B_{l}^{P}(s)$ AS AN ANALYTIC FUNCTION OF $l$}

At this point the discussion is to be specialized to the $\pi \pi$ amplitude but the general nature of the main points is evident. Suppose that for isotopic spin $I$ in the $t$ channel there is a single trajectory $\alpha_{I}(t)$ extending into the right-half $J$ plane. If the residue of this pole in $B_{l}^{I}(t)$ is $\gamma_{I}(t)$ then the usual Sommerfeld-Watson transform ${ }^{8}$ leads to a contribution to $A_{I}(t, s)$ from this pole

\footnotetext{
${ }^{25} \mathrm{It}$ is in the construction of $B_{l}{ }^{P}(s)$ that the major practical difference arises between the scheme proposed here and that of D. Wong in reference 5. The ideas underlying the two schemes are closely related.
} 
equal to

$$
\begin{aligned}
& -\pi\left[2 \alpha_{I}(t)+1\right] \gamma_{I}(t) q_{t}{ }^{2 \alpha_{I}(t)} \\
& \times \frac{P_{\alpha_{I}(t)}\left(-1-s / 2 q_{t}{ }^{2}\right)+(-1)^{I} P_{\alpha_{I}(t)}\left(1+s / 2 q_{t}{ }^{2}\right)}{2 \sin \pi \alpha_{I}(t)} .
\end{aligned}
$$

The $s$ cut in this formula begins at $s=0$, however, and recently Khuri and, independently, Jones have pointed out that the cut may be displaced by using in place of the Legendre function,

$$
\begin{aligned}
& \mathfrak{R}_{\alpha}\left(z, z_{1}\right)=\int_{z_{1}+\left(z_{1}{ }^{2}-1\right)^{1 / 2}}^{\infty} d x \frac{x^{\alpha}}{\left(1-2 z x+x^{2}\right)^{1 / 2}}, \quad \operatorname{Re} \alpha<0, \\
& =-\pi \frac{P_{\alpha}(-z)}{\sin \pi \alpha}-\int_{0}^{z_{1}+\left(z_{1}{ }^{2}-1\right) 1 / 2} d x \frac{x^{\alpha}}{\left(1-2 z x+x^{2}\right)^{1 / 2}} \\
& \operatorname{Re} \alpha>-1 \text {, }
\end{aligned}
$$

the two forms being analytic continuations of one another ${ }^{26}$ The function

$$
\begin{aligned}
R_{I}{ }^{\prime}(t, s)=\gamma_{I}(t) q_{t}{ }^{2 \alpha_{I}(t)} & {\left[2 \alpha_{I}(t)+1\right] } \\
& \times Q_{\alpha_{I}}\left(1+s / 2 q_{t}{ }^{2}, 1+s_{1} / 2 q_{t}{ }^{2}\right)
\end{aligned}
$$

can then easily be shown to satisfy a Mandelstam representation, with a real double-spectral function that is nonzero in two regions ${ }^{26}$ : (a) $s>s_{1}, q_{t}{ }^{2}>0$ and (b) $s>s_{1}$, $4 q_{t}{ }^{2}<-s_{1}$. Provided $\operatorname{Re} \alpha_{I}(t)<0$ throughout the latter region, as is guaranteed by our basic assumptions, we may subtract out the contribution of this spurious piece of double spectral function, thereby achieving an analytic function $R_{I}(t, s)$, real for $s<s_{1}, q_{t}{ }^{2}<0$, and with the same asymptotic properties as $R_{I}^{\prime}(t, s)$. This new function still has poles in $t$ when and only when $\alpha_{I}(t)$ passes through positive integers (or zero) and has no poles in $s$. All poles on or near the physical sheet are included with the correct residues, therefore, when terms of the form of $R_{I}$ for each high-ranking trajectory are superposed. There are no spurious poles. The correct asymptotic behavior within the strip also is ensured. On this basis, therefore, we use in place of (IV.1),

$$
A_{I}{ }^{P}(t, s)=\frac{1}{2}\left[R_{I}(t, s)+(-1)^{I} R_{I}(t, u)\right]
$$

as the contribution from an individual pole in the $t$ channel.

We perform the projection needed for $B_{l}{ }^{P}(s)$ in a slightly unconventional manner so as to stay within the region of reality. Normally, the continuation in angular momentum is made by the Froissart-Gribov formula ${ }^{27}$ in terms of the absorptive parts in the $t$ and $u$ channels. Wong, however, has pointed out the equivalent

${ }^{26}$ N. Khuri, Institute for Advanced Study, 1962 (unpublished); E. Jones (private communication, Berkeley, 1962); see also, G. F. Chew, University of Cambridge, 1963 (unpublished).

${ }^{27} \mathrm{M}$. Froissart, Report to the La Jolla Conference on Theoretical Physics, June, 1961 (unpublished); V. N. Gribov, J. Exptl. Theoret. Phys (U.S.S.R.) 41, 667 and 1962 (1961). representation ${ }^{28}$

$$
\begin{aligned}
q_{\mathrm{s}}{ }^{2 l} B^{( \pm)}(s, l)= & \frac{1}{2} \int_{-1}^{+1} d z P_{l}(z) A^{( \pm)}(s, z) \\
& -\frac{\sin \pi l}{\pi} \int_{-\infty}^{-1} d z Q_{l}(-z) A^{( \pm)}(s, z),
\end{aligned}
$$

where $A^{( \pm)}(s, z)$ is the pair of functions constructed to have only a right cut in the $z$ plane (with $q_{s}{ }^{2}$ positive) and whose even and odd parts in $z$, respectively, coincide with the even and odd parts of $A(s, z) .{ }^{11}$ Because of the Bose statistics in the $\pi \pi$ amplitude we may suppress the $( \pm)$ superscript and the required projection of (IV.4) simply acquires a factor 2 altogether with the crossing matrix

$$
\begin{aligned}
B_{I} P(s, l) & =\sum_{I^{\prime}} \beta_{I I^{\prime}} \frac{1}{q_{s}^{2 l+2}} \\
\times & \left\{\frac{1}{2} \int_{-4 q_{s}{ }^{2}}^{0} d t P_{l}\left(1+\frac{t}{2 q_{s}}\right) A_{I^{\prime}}{ }^{P}(t, s)\right. \\
& \left.-\frac{\sin \pi l}{\pi} \int_{-\infty}^{-4 q_{q^{2}}} d t Q_{l}\left(-1-t / 2 q_{\mathrm{s}}{ }^{2}\right) A_{I^{\prime}}{ }^{P}(t, s)\right\} .
\end{aligned}
$$

Here

$$
\beta_{I I^{\prime}}=\left(\begin{array}{rrr}
\frac{1}{3} & 1 & \frac{5}{3} \\
\frac{1}{3} & \frac{1}{2} & -\frac{5}{6} \\
\frac{1}{3} & -\frac{1}{2} & \frac{1}{6}
\end{array}\right) .
$$

The domain of holomorphy in $l$ of $B_{I}^{P}(s, l)$ is evidently determined by the behavior of $A_{I^{\prime}}{ }^{P}(t, s)$ at large (negative) $t$. If all the $\alpha_{I^{\prime}}(t)$ approach finite limits at $\infty$ we may read off this behavior from (IV.4) and (IV.3) as

$$
A_{I^{\prime}}{ }^{P}(t, s) \underset{t \rightarrow \pm \infty}{\propto} t^{\alpha I^{\prime}(\infty)} \ln t \gamma_{I^{\prime}}(t) \times(\text { function of } s) \text {. }
$$

The reduced residue $\gamma_{I}(t)$ for the leading trajectory approaches a constant as $t \rightarrow \infty$ in potential scattering. If such is true in general, we see that $B_{I}{ }^{P}(s, l)$ is holomorphic for

$$
\operatorname{Re} l>\max \alpha_{I}(\infty) \text {. }
$$

Evidently, if all the $\alpha_{I}(t)$ approach the same limit at infinity (perhaps -1 , as suggested by the work of Gribov $^{16}$ ) we have a self-consistent situation. There is no reason to expect such a degree of consistency in the strip approximation; outside the strips our equations unreliable. Nevertheless, if all $\alpha_{I}(\infty)$ are negative, as originally supposed, we have a chance of finding approximately consistent solutions of our equations across the strips.

Notice that as the equations are formulated one deals only with the trajectories $\alpha_{I}(t)$ for $t<0$, which means that the self-consistency problem only involves real

${ }^{28} \mathrm{D}$. Wong (private communication, 1962). 
$l \leqslant 1$. Hopefully, the parameter $s_{1}$ can be determined by the maximum strength requirement $\alpha_{I=0}(t=0)=1$ and, as argued in the following section, the other trajectories automatically fall below the Pomeranchuk. Thus, we hope to satisfy the Froissart limit, even though unitarity at high energies is not explicitly invoked. It is not so hopeful that we can consistently satisfy the requirement of $\gamma_{I=0}(t)$ vanishing at that negative $t$ where $\alpha_{I=0}=0$. Could unitarity be explicitly enforced such a result would be guaranteed, but we must depend on some not yet understood mechanism to produce a zero in the numerator function $N_{I=0}$ at the appropriate place. Nevertheless, this deficiency is not as disastrous as might appear at first sight because there is no prospect in any event of finding a mathematically self-consistent solution to our bootstrap problem. What will be done is to assume a set of $\alpha_{I}(t)$ and $\gamma_{I}(t)$ for $t<0$ and see how closely these reproduce themselves. The trial functions $\alpha_{I}(t)$ and $\gamma_{I}(t)$ can be characterized through effective range formulas by a finite number of adjustable parameters over the range where $\alpha_{I} \gtrsim 0$ (see following section) and it will be possible to constrain the formula for $\gamma_{I=0}(t)$ so that this function vanishes when $\alpha_{I=0}(t)$ vanishes, regardless of the choice of adjustable parameters. It should be extremely interesting to see whether the $\gamma_{I=0}(t)$, emerging from the solution of our $N / D$ equations, shows any tendency to vanish in the appropriate place. Such a vanishing could result from a short-range repulsive force together with a longer range attraction, the combination producing a zero in $N_{I=0}(s, l)$. That a short-range repulsion systematically should accompany strongly attractive long-range forces has been argued previously, ${ }^{10}$ but even if our current strip equations contain manifestations of this repulsion there is no visible reason why the zero in $N_{I-0}(s, l)$ must occur at precisely the correct point. Explicit satisfaction of unitarity in $s$ and $t$ channels simultaneously is presumably required to achieve such a result.

\section{EFFECTIVE-RANGE FORMULAS FOR TRAJECTORIES AND RESIDUES}

In the region $t<0$ where $\alpha(t)$ and $\gamma(t)$ are real and free from singularities it should be possible to approximate these functions by effective-range formulas of the type suggested by Balàzs. ${ }^{29}$ With nonintersecting trajectories [simple zeros of $D(t, l)]$ the previous discussion allows us to start from the general forms

$$
\begin{aligned}
& \alpha(t)=a_{1}+\int_{t_{0}}^{\infty} d t^{\prime} \frac{\rho_{\alpha}\left(t^{\prime}\right)}{t^{\prime}-t} \\
& \gamma(t)=c_{1}+\int_{t_{0}}^{\infty} d t^{\prime} \frac{\rho_{\gamma}\left(t^{\prime}\right)}{t^{\prime}-t}
\end{aligned}
$$

where $a_{1}, c_{1}, \rho_{\alpha}(t)$, and $\rho_{\gamma}(t)$ are real. Balàzs has analyzed this type of function ${ }^{29}$ and shown that, to achieve a

\footnotetext{
${ }^{29}$ L. Balàzs, Phys. Rev. 125, 2179 (1962).
}

prescribed accuracy over a prescribed interval away from the cuts, one may determine on an $a$ priori basis the number and the location of poles which replace the cuts. Often two poles lead to reasonable accuracy, but each situation must be individually analyzed. Here we want to represent our functions over the interval $-s_{1} \leqslant t<0$, so the number of poles depends on the ratio $s_{1} / t_{0}$, which may be large. However, the discontinuities $\rho_{a, \gamma}(t)$ each start from threshold like $\left(t-t_{0}\right)^{\alpha\left(t_{0}\right)+1 / 2}$, so the effective threshold is displaced upward to some reasonable fraction of $s_{1}$ in the interesting cases. It is plausible, then, to expect a two-pole representation of the cut generally to suffice.

Our effective-range formulas with two poles would be

$$
\begin{aligned}
& \alpha(t)=a_{1}+a_{2} /\left(t_{2}-t\right)+a_{3} /\left(t_{3}-t\right), \\
& \gamma(t)=c_{1}+c_{2} /\left(t_{2}-t\right)+c_{3} /\left(t_{3}-t\right),
\end{aligned}
$$

where $t_{2}$ and $t_{3}$ are to be chosen by a Balàzs type of analysis and lie in the range $t_{0}<t_{2}, t_{3} \leqslant s_{1}$. If $\alpha(t)$ has a zero for $t<0$ for a trajectory of even $J$ signature (such as the Pomeranchuk trajectory) we determine one of the $c$ parameters by the requirement that $\gamma(t)$ have a zero at the same point. It may be that $a_{1}$ should be fixed at -1 if further investigation shows this to be the asymptotic limit of all trajectories. For the Pomeranchuk trajectory a second parameter in (V.3) may be fixed by the maximum strength requirement $\alpha(0)=1$.

A check on the adequacy of the forms (V.3) and (V.4) is provided by the actual solutions of our $N / D$ equations; it will soon be evident whether more poles should be added. One also learns whether in our strip approximation $\alpha(\infty)=-1$ and whether the zeros of $D(t, l)$ are, in fact, simple for the top-ranking trajectories, as we have assumed.

\section{PION-PION DYNAMICS}

We conclude with an optimistic forecast of how the calculations of the $\pi \pi$ amplitude in the one-channel strip approximation may develop. Our prediction is based not only at a peek at nature but on many previous calculations, ${ }^{30}$ particularly those of Chew and Mandelstam, ${ }^{1}$ of Ball and Wong, ${ }^{31}$ of Zachariasen and Zemach, ${ }^{24}$ of Wong, ${ }^{5}$ and of Balàzs. ${ }^{32}$

A natural starting point would be to assume just the Pomeranchuk trajectory and look for an approximately self-consistent solution involving the $I=0$ amplitude alone. We predict that no such solution will be found coming close to the maximum strength requirement $\alpha_{I=0}(t=0)=1$. The reason is the relatively small coefficient $\frac{1}{3}$ coupling the $I=0$ state to itself in the crossing matrix (IV.9), plus the even $J$ signature of the Pomeranchuk trajectory which makes its (real) contribution

${ }^{30} \mathrm{~A}$ great many $\pi \pi$ calculations, in addition to those listed, have been published but employ methods that are difficult to relate to the calculation proposed here.

31 J. Ball and D. Wong, Phys. Rev. Letters 7, 390 (1961). 
to (IV.4) small when $\alpha_{I=0}$ is near 1. (It is the imaginary part of $A_{I=0}{ }^{P}$ that is dominant at high energy.) The force, in question, is attractive and one might think that $\gamma_{I=0}(t)$ could become large enough to counterbalance the aforementioned effects, but we expect from past experience that the analytic continuation of unitarity in the $t$ channel, through the $N / D$ equations, constrains the magnitude of $\gamma_{I=0}(t)$.

One then looks for help from either $I=1$ or $I=2$ and a glance at the crossing matrix plus consideration of $J$ signature shows the former to be the more promising. We, therefore, try adding an $I=1$ trajectory, which according to (IV.7) must lie below the Pomeranchuk trajectory because the attraction it feels from $I=1$ exchange is only half as strong. Now there is a chance for a fairly consistent solution (see the work of Balàzs $\mathrm{s}^{22}$ ), where $A_{I=1}{ }^{P}$ delivers a strong attraction to $I=0$, a weaker but still attractive force for $I=1$ and a repulsion for $I=2$. The uniform attraction delivered to all three states by $A_{I=0}{ }^{P}$ is relatively weak as we have seen, so the net force acting in $I=2$ is probably repulsive with the trajectory here staying in the left-half $J$ plane.

We hope that the results are not too sensitive to the precise value of $s_{1}$ and that, perhaps, this parameter can be fixed by requiring $\alpha_{I=0}(t=0)=1$ (the results of Balàzs support such a hope $\mathrm{e}^{32}$ ). A difficult test of the theory is to look for second-rank trajectories. Current experimental indications are that at least one such reaches the right-half $J$ plane for $I=0$ but probably none for $I=1$. Since our force strength is at a maximum for $I=0$ the situation is hopeful, but no previous calculations throw any light on this matter.

In view of the one-channel approximation, we are not optimistic about the accuracy of predicted residues $\gamma_{I}(t)$, although normalizing the Pomeranchuk trajectory at $t=0$ may lead to reasonable predictions of resonance energies. ${ }^{33}$ According to the estimates of Blankenbecler ${ }^{34}$ and of Zachariasen and Zemach, ${ }^{24}$ it is necessary to include at least the $\pi \omega$ channel in addition to the $\pi \pi$ if accurate resonance widths are to be achieved.

\footnotetext{
${ }^{33}$ Although the self-consistent problem is restricted to $t<0$, there is no difficulty in continuing our solutions to $t>t_{0}$ in order to see if resonances are predicted and with what masses and widths. ${ }^{34}$ R. Blankenbecler, Phys. Rev. 125, 755 (1962).
}

To emphasize the content of the particular enterprise under consideration we summarize some obvious points of contact with experiment :

(1) The function $\alpha_{I=0}(t)$ for $-s_{1} \leqslant t \leqslant 0$ already has been roughly measured from high-energy $p p$ elastic scattering. ${ }^{35}$

(2) The residue $\gamma_{I=0}(t=0)$ is known accurately from total cross-section measurements. ${ }^{36}$

(3) A prediction emerges from the calculation as to whether a $J=2, I=0$ resonance is to be expected and with what mass and width.

(4) The measured mass of the $\rho$ tells us that $\operatorname{Re} \alpha_{I=1}\left(t=30 m_{\pi}^{2}\right)=1$.

(5) The residue $\gamma_{I=1}\left(t=30 m_{\pi}^{2}\right)$ is known roughly from the width of the $\rho$.

(6) The value of $\alpha_{I=1}(t=0)$ is known roughly from the energy dependence of the $\pi^{+} p$ and $\pi^{-} p$ total cross-section difference. ${ }^{37}$

(7) The height of the secondary trajectory for $I=0$ is known at $t=0$ to be $\sim 0.5 .^{38}$

(8) It is indicated experimentally that no secondary trajectory for $I=1$ and no trajectories at all for $I=2$ reach the right-half $J$ plane.

All these points and, no doubt, others to emerge from future experiments lie within the domain of our bootstrap calculation. All are supposed to be predicted, starting only from a knowledge of the pion mass and quantum numbers. Of course, as emphasized repeatedly in this paper, we do not expect accurate predictions until all the important two-body channels are included.

\section{ACKNOWLEDGMENTS}

Helpful discussions with J. Ball, W. Frazer, S. Frautschi, F. Low, S. Mandelstam, K. Wilson, and D. Wong are gratefully acknowledged. I wish also to express thanks to L. Van Hove for the hospitality of the CERN Theoretical Study Division, where this paper was written.

${ }^{35}$ A. N. Diddens, E. Lillethum, G. Manning, A. E. Taylor, T. G. Walker, and A. M. Wetherell, Phys. Rev. Letters 9, 111 (1962).

${ }^{36}$ M. Gell-Mann, Phys. Rev. Letters 8, 263 (1962); V. N. Gribov and I. Pomeranchuk, ibid. 8, 343 (1962).

${ }_{37}$ B. M. Udgaonkar, Phys. Rev. Letters 8, 142 (1962).

${ }^{38}$ K. Igi, Phys. Rev. Letters 9, 76 (1962). 